Diabetologia 10, 541-548 (1974)

(C) by Springer-Verlag 1974

\title{
Segmental Demyelination in the Distal Peripheral Nerves of Chronically Diabetic Chinese Hamsters
}

\author{
W.W. Schlaepfer, G.C. Gerritsen, and W.E. Dulin \\ Department of Patholog'y, Washington University Medical School, St. Louis, Missouri and the Upjohn Company, \\ Kalamazoo, Michigan, USA.
}

Summary. Distal tibial nerves of 14 chronically diabetic and 6 control Chinese hamsters were examined using ultrastructural and teased whole-mount methods. Glucosuria was present for varying intervals up to 976 days, while 10 hamsters manifested ketonuria for intervals up to 551 days. Myelin internodal ensheathment patterns were analyzed from whole-mount preparations. A normal proportional relationship existed between myelin internodal length and fiber size in the peripheral nerves from control animals. Patterns of segmental demyelination. were seen in the internode length/fiber size relationships of experimental animals. These changes were manifested by increased disparity of internode lengths along individ. ual medullated fibers as well as by a generalized decrease of internode lengths, especially along large fibers. In addition, occasional fibers were seen undergoing acute axonal (Wallerian) degeneration in teased fiber preparations of diabetic animals. Variations in severity of structural alterations correlated with the length of diabetic state and were markedly increased in some animals with ketonuria. Examinations of some proximal sciatic nerves gave indications of a marked proximo-distal gradient in the manisfestations of the structural alterations in peripheral nerve. Electron microscopic evidence of demyelinative and remyelinative phenomena could be seen in diabetic hamsters. Both light and electron raicroscopic changes in diabetic animals were similar to those reported in diabetic neuropathy.

Key words: Diabetes, hamster, neuropathy, teasedfiber, ultrastructure, demyelination, axonal degeneration, onion-bulb, nerve fiber, peripheral nerve.
The spontaneous diabetes which occurs in an inbred strain of Chinese hamsters may well serve as a useful model for the study of complications of the diabetic state. Structural alterations such as degenerative changes in the beta cells in the islets of Langerhans [1], renal glomerulopathy [2], and retinal microangiopathy [3] with extravasational deposits [4] have been shown to occur in these diabetic animals. This study demonstrates a segmental demyelination of peripheral nerves in the chronically diabetic Chinese hamster, an alteration which is most pronounced in the distal nerve segments and is aggravated by the concomitance of ketotic conditions in the animal.

\section{Material and Methods}

20 animals were available for study, including 14 with chronic diabetes and 6 age-matched controls. 10 diabetic hamsters had longstanding ketosis as evidenced by prolonged ketonuria. Sciatic and tibial nerves were fixed by excision and immersion or by animal perfusion techniques. Ultrastructural studies were conducted on nerve segments fixed in a buffered solution containing a mixture of glutaeraldehyde and paraformaldehyde. Optimum fixation was achieved by perfusion fixation with $1 \%$ concentrations of aldehydes in $0.05 \mathrm{M}$ cacodylate buffer with $2.5 \mathrm{mM}$ $\mathrm{CaCl}_{2}$ followed by immersion in buffered solutions containing $2 \%$ concentrations of aldehydes. Subsequently, the tissues were osmicated, stained en bloc with uranyl acetate, dehydrated and embedded in epoxy resin. Thin sections were stained with lead citrate before examination in a Siemens Elmskop I.

Whole-mount teased preparations of nerve were conducted on nerve segments fixed in $10 \%$ buffered formalin for $4 \mathrm{hrs}$, washed and stored in buffered solutions and subsequently osmicated. Separation of fibers could be facilitated by a $30-60$ min treatment of osmicated nerve in $30 \%$ glycerol. Teasing operations were performed with very fine eye forceps under a dissecting microscope and the entire fiber complement of representative fascicles were separated and aligned in parallel rows on microscopic slides before coverslipping and examination by light microscopy. Measurements were conducted by direct visualization using a precalibrated linear marker in the eyepiece of the microscope. Nerve fibers with 3 or more clearly visualized consecutive myelin internodes were selected for measurement. Nerve fiber diameters varied along individual fibers, but maximum measurements were used. Myelin internodal lengths could be measured with much greater reproducibility.

Graphs showing the myelin internode lengths/fiber diameter were constructed as originally devised by Fullerton et al. [5]. Measurements from diabetic and control animals were treated in an identical fashion. Fibers with maximum diameters measured in nearest micron integer were charted within one micron distance of that value (e.g. a $7 \mathrm{\mu m}$ fiber could be placed between the $6 \mathrm{um}$ and $8 \mu \mathrm{m}$ values on the abscissa). Within these limitations the sequential arrangement of fibers in the graphs were arrayed according to the size of their composite myelin internode lengths. In 
this manner some compensation could be given to the variance in fiber diameter measurements relative to the highly reproducible values of myelin internode lengths.

\section{Results}

Whole-Mount Teased Preparations

Comparative myelin internodal patterns in reationship to fiber diameters in the distal tibial nerves of control, diabetic and diabetic-ketotic Chinese hamsters are illustrated in Fig. 1. Control tibial nerves displayed the normal proportional relationship between fiber size and internode length, a feature of peripheral nerve reflecting the disproportional elongation of myelin sheaths during growth [6]. In the diabetic animals, the normal myelin internodal pattern was disrupted. Two types of changes were observed: First, there was an overall reduction in the lengths of myelin internodes, which was predominantly mani-
Control

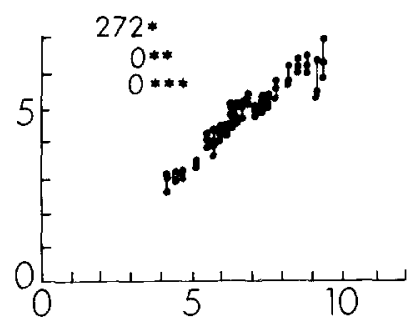

$277 *$
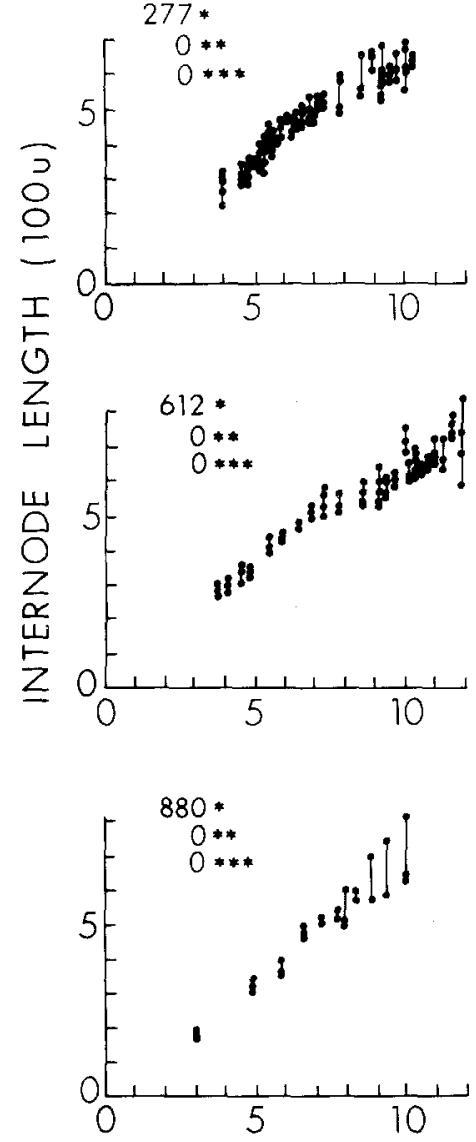

Diabetic
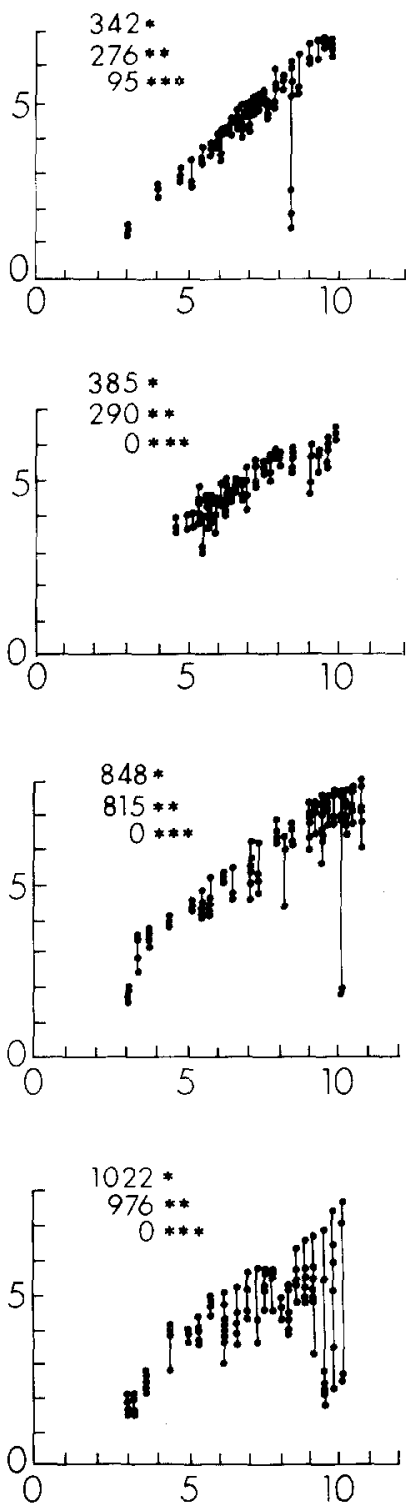

FIBER DIAMETER (u)
Diabetic - Ketotic
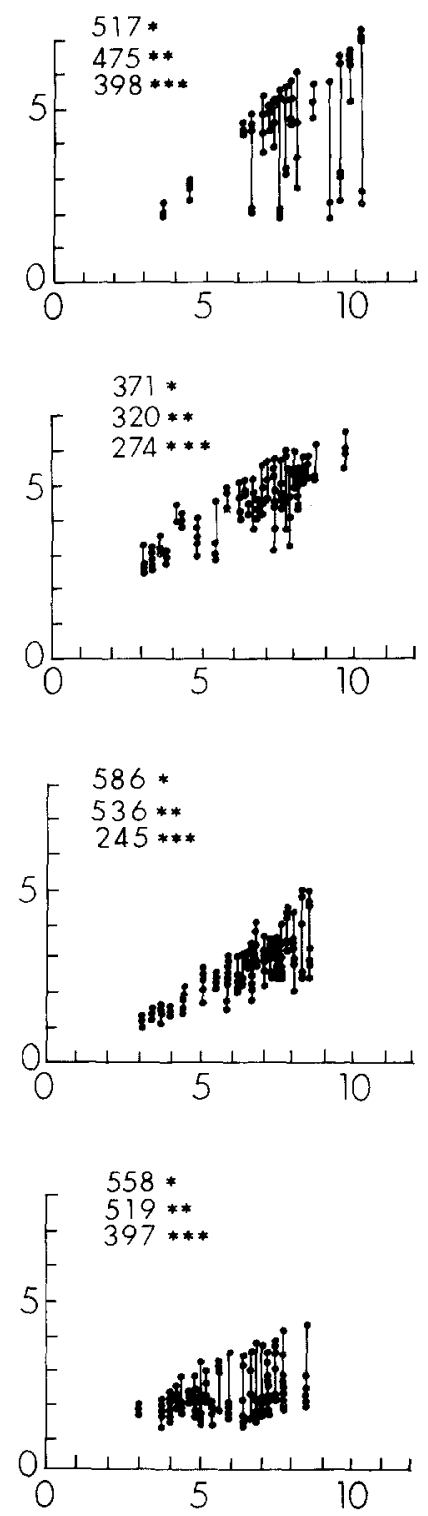

Fig. 1. Lengths of myelin internodes in relationship to nerve fiber diameters in distal tibial nerves of control, diabetic and diabetic-ketotic Chinese hamsters. Each vertical bar interconnects measured internode lengths (dots) along an, individual nerve fiber. Each graph represents the measurements of several representative nerve fibers from one animal with animal age $(*)$, period of glycosuria $(* *)$ and period of ketonuria $(* * *)$ measured in days 
fested among the larger myelinated fibers. Second, there appeared to be a greater discrepancy in lengths of myelin internodes along individual fibers. Both of these alterations in myelin internodes increased in severity with prolongation of the diabetic state and with the accompanying presence of ketonuria. Similar findings were present in the distal nerves of the animals not depicted in Fig. 1. would be expected from remyelination following focal demyelination or nerve regeneration. Some fibers were encountered with multiple small myelin internodes $(200-300 \mu \mathrm{m})$ which could represent segments of regenerated or remyelinated nerve fibers. A second abnormality of myelin ensheathment of diabetic animal nerves was a fragmentation of myelin indicative of axonal (Wallerian) degeneration along the pre-
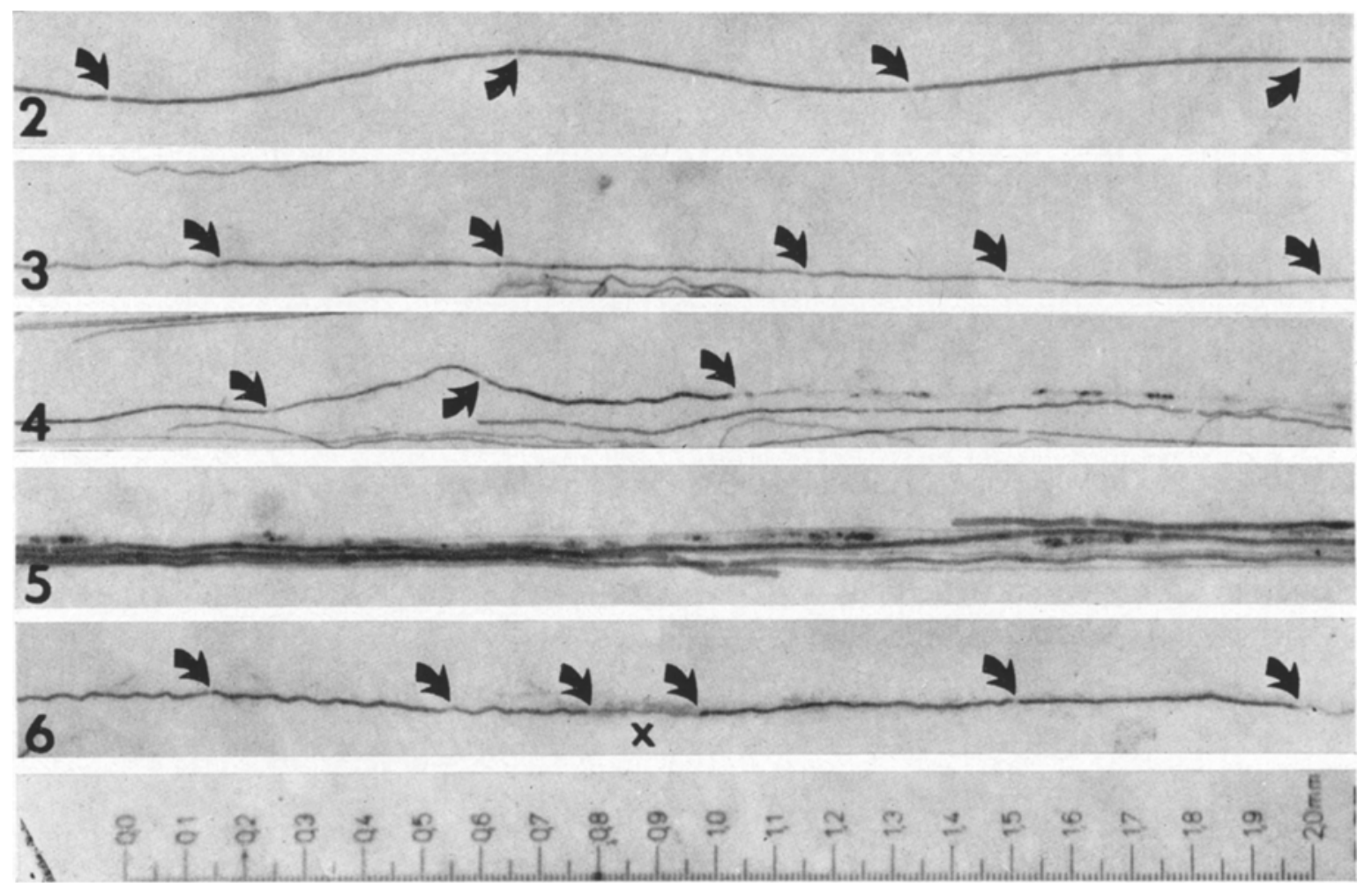

Figs. 2-6. Whole-mount, teased preparations of distal tibial nerves demonstrating consecutive myelin internodes between nodes of Ranvier (arrows). Fig. 2 shows myelin internodes of uniform length and thickness along nerve fiber from control hamster. Fig. 3 reveals foreshortened myelin internodes of disparate lengths but uniform thickness' along nerve fiber from diabetic-ketotic animal. Fig. 4 shows fragmentation of distal myelin sheaths indicative of axonal degeneration in continuity with foreshortened myelin internodes of nerve fiber from diabetic hamster. Fig. 5 shows small cluster of myelinated fibers from diabetic animal containing a single fiber which displays a fragmentation of its myelin sheath throughout the entire examined segment. Fig. 6 demonstrates a thin and short intercalated myelin internode $(x)$ interspersed between thicker myelin internodes. Magnification is indicated by micrometer scale (bottom) which has been photographed and enlarged to the same extent as the whole-mount, teased preparations, excepting Fig. 5 which has been enlarged another $40 \%$

Characteristic myelin ensheathment along tibial nerves of control animals revealed a sequence of myelin sheaths of uniform length and thickness (Fig. 2). Slight variations of intranodal gaps and terminal configurations of myelin sheaths were noted, but not quantitated. In diabetic animals three types of altered patterns of myelin ensheathment were seen. The most common abnormality was an increasing discrepancy in lengths of myelin internodes of approximately equal thickness (Fig. 3). The multiple foreshortened internodes along these fibers had a random distribution and were often greater in length $(400-600 \mu \mathrm{m})$ than sumptive distal portion of a fiber showing discrepant myelin internodal lengths along the adjacent intact portion of fiber (Fig. 4). More often, individual degenerating nerve fibers were encountered which showed fragmentation of myelin indicative of axonal degeneration along the entire portion of examined nerve segment (Fig. 5). Fibers undergoing axonal degeneration were sporadically encountered among the diabetic animals, their incidence estimated at $0-3 \%$ of myelinated fiber populations. The third alteration of myelin ensheathment of nerves from diabetic animals was the presence of short and thin 
myelin internodes interspersed between thicker and longer myelin internode segments (Fig. 6). These short internodes were characteristic of intercalated internodes [7] and accordingly, probably represented remyelinated segments of axon. The remaining thicker myelin internodes along these fibers were often disparate in their internodal lengths. Consistent and characteristic alterations of intranodal gaps or para-

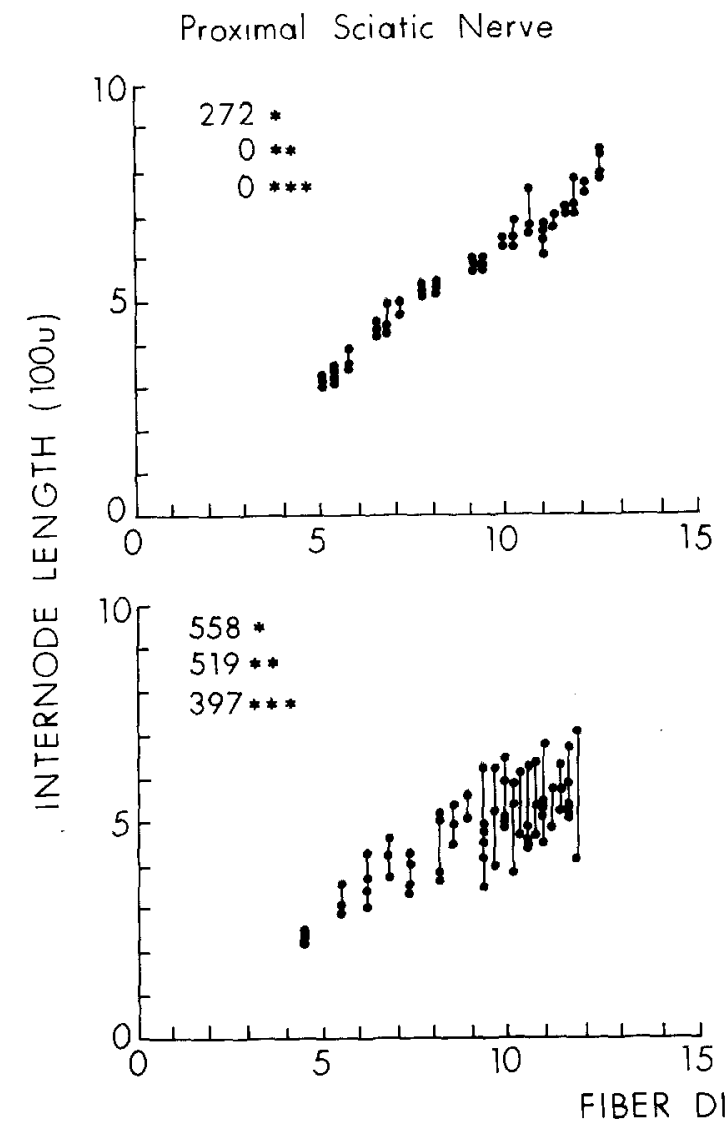

experimental diabetic nerve. The myelinated and unmyelinated nerve fibers from the control animals and the large majority of these nerve fiber populations from diabetic animals appeared unremarkable. However, occasional myelinated fibers from diabetic animals revealed distinctive abnormalities. Fig. 8 illustrates the paranodal region of a fiber with axoplasmic accumulation of neurofilaments and assorted vesicular-
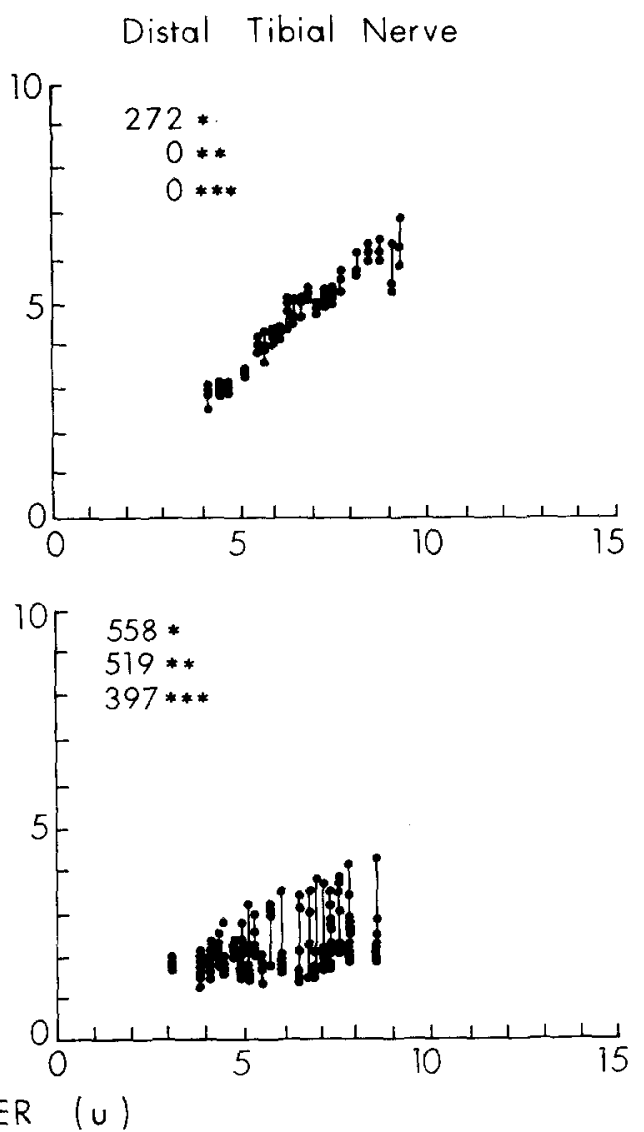

Fig. 7. Lengths of myelin internodes in relationship to nerve fibers diameters in proximal sciatic and distal tibial nerves of control and diabetic-ketotic hamsters. See Fig. 1 for explanation of symbols

nodal myelin architecture were not convincingly apparent in the diabetic animals.

Myelin ensheathment patterns of proximal nerve segments taken from the sciatic nerve at the sciatic notch were compared with ensheathment patterns of the distal tibial nerves in some severly altered experimental animals (Fig. 7). These studies indicated a marked proximo-distal gradient in the severity of abnormal myelin ensheathment. The marked alteration of myelin internodal ensheathment patterns were clearly evident only in the distal segments.

\section{Ultrastructural Examinations}

Representative distal tibial nerves of control and experimental animals were examined in an attempt to characterize some of the qualitative changes in the
Figs. 8-11. Fig. 8. Transverse section of myelinated fiber from diabetic-ketotic hamster showing axoplasm (A) with increase of neurofilaments and tubular-vesicular structures. The terminus of the myelin sheath of this fiber appears retracted and the composite myelin lamellae therein terminate in linear or punctate densities. $\times 12,000$. Fig. 9. Transverse section of distal tibial nerve from diabetic hamster showing central myelinated fiber encircled by a thin Schwann cell process, a feature indicative of "onion-bulb" formation or hypertrophic change. $\times 8,000$. Fig. 10. Transverse section of a denuded axon (A) within the cytoplasm of a Schwann cell (SC). Lipid debris from the myelin sheath may be seen in an adjacent macrophage (M) of the distal tibial nerve from a diabetic-ketotic animal. $\times 6,000$. Fig. 11. Transverse section of an endoneurial blood vessel from a diabetic-ketotic hamster containing an erythrocyte (RBC) and surrounded by a macrophage (M) with lipid debris. The intervening basement membrane appears thickened and reduplicated. 

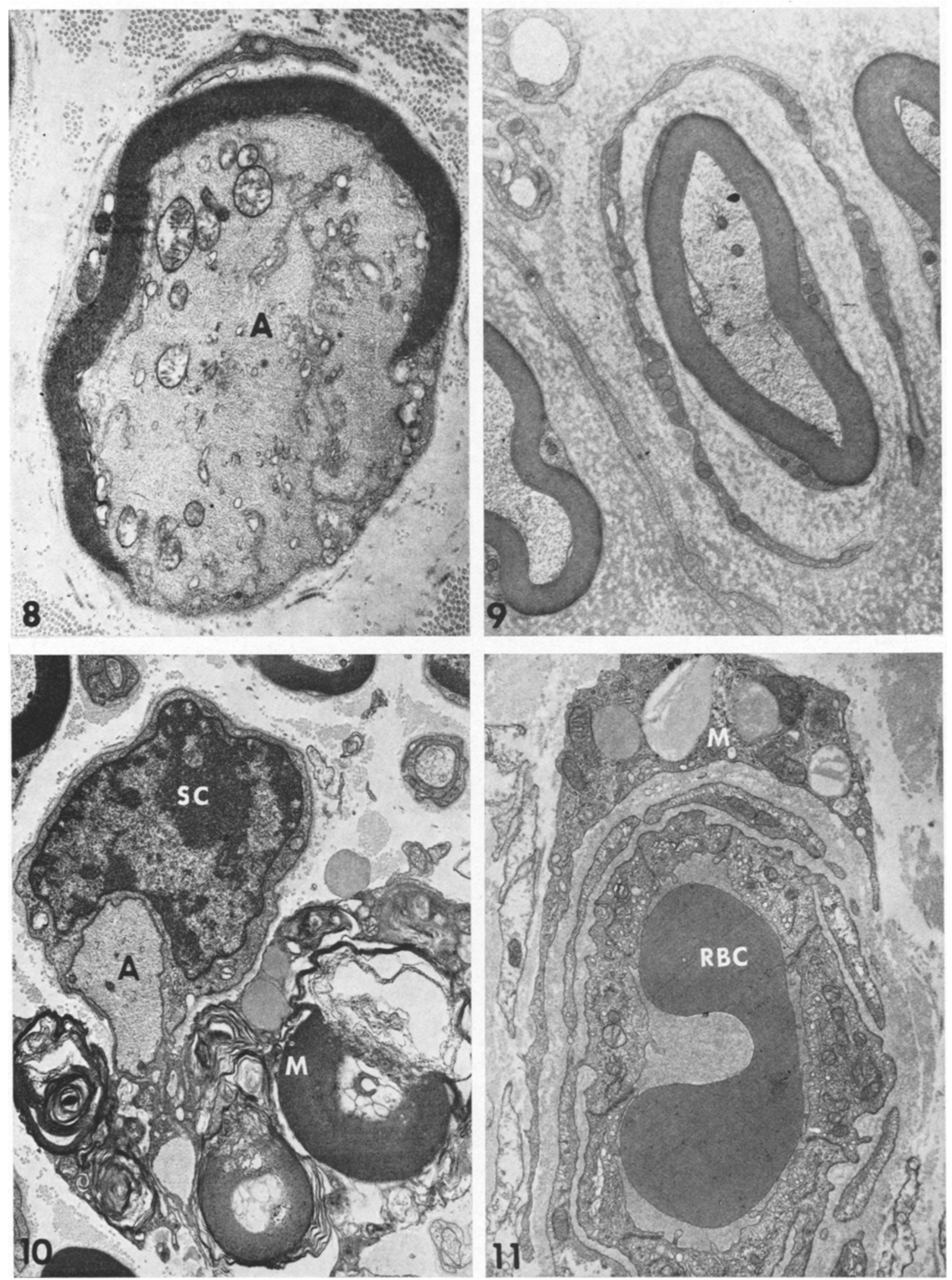
tubular structures and apparent retraction of the myelin sheath. Of particular interest are the ends of the myelin lamellae which appear to terminate in electron dense linear or punctate structures which are continuous with the major dense lines of each lamella. The normal loops of Schwann cell and intercellular appositional apparatus [8] is not apparent.

Occasional fibers demonstrated active demyelinative changes which was sometimes characterized by a macrophage filled with myelin debris immediately adjacent to an intact but denuded axon (Fig. 10). More frequent evidence of demyelination and remyelination was seen in the form of lamellar encirclement of myelinated axons by thin Schwann cell processes (Fig. 9), a change characteristic of "onion-bulb" or hypertrophic alterations, indicative of non-specific remyelinative phenomena $[9,10]$.

Some alterations were noted in the endoneurial microvasculature of the experimental animals. Most frequently evidence of lipid debris could be seen in perivascular macrophages (Fig. 11). Some of these vessels also displayed some reduplication and possible thickening of their basement membranes (Fig. 11). No attempt was made to quantitate these changes.

\section{Discussion}

The demonstration of degenerative structural alterations in the peripheral nerves of chronically diabetic Chinese hamsters represents a significant advance in the search for an experimental model of diabetic neuropathy. Segmental demyelination has been reported in alloxan-induced diabetic rats $[11,12]$; however, the findings of these studies have not yet been published. Variations in nodal myelin architecture in alloxan-diabetic animals $[13,14]$ are less convincing manifestations of segmental demyelination. Similar variations in nodal myelin configurations were noted in both our control and diabetic animals. Their significance remains therefore unclear and could be appreciated only by accurate quantitative analyses. Moreover, Thomas [15] demonstrated a lack of structural alterations in the peripheral nerves of chronic streptocytozin-induced diabetic rats although subacute reduction of conduction velocity was observed in these same nerves. This had previously been reported to occur in alloxan-induced diabetic peripheral nerve as well [12, 16, 17]. More convincing evidence of structural alterations in the peripheral nerves of experimental diabetic animals is the alterations in subneural apparatus and terminal nerve branching patterns with alloxan hyperglycemia [13]. It is of interest that these changes were detected in the distalmost regions of the peripheral nervous system. Studies of structural and distributional changes of peripheral nerves in the human diabetic condition have shown that the degenerative changes are predominantly manifested in the distal portions of peri- pheral nerves and that the preponderance of change occurs in the sensory and autonomic peripheral nerve systems. Observations which have lead to these conclusions include the preferential loss of myelinated nerve fibers, characteristic of human diabetic neuropathy [18], in more distal nerve segments [19-22], the selective degradative changes in posterior nerve roots relative to their anterior root counterparts $[18,20,21,23]$ and the preponderance of peripheral nerve cell loss within the sensory $[20,23]$ and autonomic [23-25] ganglia. The peripheral motor nerve system is also involved in human diabetic neuropathy; however, clear-cut structural alterations of this system are largely limited to the distal innervative areas, manifested by degeneration and regeneration of terminal nerves $[26,27]$, alterations of subneural apparatus $[26,27]$ and "single fiber" as well as "fiber group" muscle atrophy [27, 28].

More extensive studies of nerve segments from diabetic patients have revealed extensive segmental demyelination and remyelination along the composite myelinated fibers $[22,29,30]$. In addition, these studies with teased nerve preparations have revealed the concomitance of fibers undergoing axonal (Wallerian) degeneration $[29,30]$. Furthermore, quantitative analyses of fiber populations of these nerves have shown a marked reduction in the number of myelinated fibers [18]. Purely demyelinative experimental lesions of peripheral nerve as exemplified by diphtheritic neuropathy do not lead to a significant reduction of myelinated fibers $[31,32]$.

Recently, the original concept that manifest demyelination is solely an expression of Schwann cell impairment has been challenged. Dyck et al. [33] and Thomas et al. [34] have attributed demyelinative patterns of uremic neuropathy to neuronal-axonal injury. Thomas [35] has also listed several human neuropathies of reputed primary neuronal-axonal impairment in which segmental demyelination occurs. It thus seems likely that the totality of structural changes within the peripheral nervous system may more fully reveal the nature of neuropathic alterations in various dysfunctional conditions.

The changes noted in the peripheral nerves of Chinese hamsters with chronic diabetes closely resembles alterations reported in human diabetic nerves, including segmental demyelinative changes [22, 29, 30] accompanied by occasional fibers undergoing axonal (Wallerian) degeneration [29, 30]. The ultrastructural manifestations of remyelinative phenomena which are characterized by "onion-bulb" formation (Fig. 9) have also been noted in nerve biopsies from diabetic patients [36. 37]. The illustration of active transfer of myelin debris to a macrophage (Fig. 10) is probably a rather fortuitous finding of a non-specific process in the degradation of myelin debris,

While the nature of peripheral nerve changes is quite similar in diabetic human and Chinese hamster 
nerves, their distribution within the diabetic animals is of special interest. In particular, the nerve degeneration in diabetic animals was much more pronounced in the distal portions. This feature of experimental neuropathy may be related to species differences, especially considering the enormous differences in lengths of axonal extensions from the respective peripheral neurons of approximately the same size. Furthermore, these findings should alert investigators of other peripheral neuropathies to examine the distalmost extensions of nerve in small laboratory animals.

The accentuation of neuropathic structural alterations in chronically diabetic animals with ketonuria remains to be clarified. It is quite possible that ketonuria represents the manifestations of a more profound state of diabetes which would also tend to cause greater neuropathic complications. It is known that renal failure with uremia may also lead to peripheral neuropathy $[33,34,38]$ not unlike that seen in the chronic diabetic animals. Whether ketotic conditions themselves may aggrevate an underlying neuropathy is unknown. A further understanding of this relationship between diabetic neuropathy and ketoacidosis is clearly desirable.

Acknowledgements. This work was in part supported by U.S.P.H.S. Grant NS.08620 and Research Career Development Award NS-70037 to W.W.S. from the National Institutes of Health, Washington D.C., U.S.A.

\section{References}

1. Luse, S.A., Caramia, F., Gerritsen, G., Dulin, W.E.: Spontaneous diabetes mellitus in the Chinese hamster: An electron microscopic study of the Islets of Langerhans. Diabetologia 3, 97-108 (1967)

2. Shirai, T., Welsh, G.W., Sims, E.A.H.: Diabetes mellitus in the Chinese hamster. II. The evolution of renal glomerulopathy. Diabetologia 3, 266-286 (1967)

3. Federman, J.L., Gerritsen, G.C.: The retinal vasculature of the Chinese hamster: A preliminary study. Diabotologia 6, 186-191 (1970)

4. Soret, M.G., Dulin, W.E., Gerritsen, G.C.: Microangiopathy in animals with spontaneous diabetes. Advanc. Metab. Disord. 2, (Suppl. 2) 291-298 (1973)

5. Fullerton, P.M., Gilliatt, R.W., Lascelles, R.G., Morgan-Hughes, J.A.: The relation between fiber diameter and internodal length in chronic neuropathy. J. Physiol. (Lond.) 178, 26-28 (1965)

6. Schlaepfer, W.W., Myers, F.K.: Relationship of myelin internode elongation and growth in the rat sural nerve. J. comp. Neurol. 147, 255-266 (1973)

7. Lubinska, L.: "Intercalated" internodes in nerve fibers. Nature (Lond.) 181, 957-958 (1958)

8. Peters, A., Palay, S.L., Webster, H. de F.: The Fine Structure of the Nervous System. The Cells and Their Processes. p. 98-101. New York: Hoeber Medical Division of Harper and Row 1970

9. Thomas, P.K., Lascelles, R. G.: Hypertrophic neuropathy. Quart. J. Med. 36, $223-238$ (1967)

10. Webster, H. deF., Schroder, J.M., Asbury, A.K., Adams, R.D.: The role of Schwann cells in the formation of "onion-bulbs" found in chronic neuropathies. J. Neuropath. exp. Neurol. 26, 276-299 (1967)
11. Preston, G.M.: Peripheral neuropathy in the alloxandiabetic rat. J. Physiol. (Lond.) 189, 49-50 (1967)

12. Lovelace, R.E.: Experimental neuropathy in rats made diabetic with alloxan. Electroenceph. clin. Neurophysiol. 25, 399a (1968)

13. Hildebrand, J., Joffroy, A., Graff, G., Cöers, C.: Neuromuscular changes with alloxan hyperglycemia. Electrophysiological, biochemical and histological study in rats. Arch. Neurol. (Chic.) 18, 633 - 641 (1968)

14. Seneviratne, K. N., Peiris, O.A.: The effects of hypoxia on the excitability of the isolated peripheral nerves of alloxan-diabetic rats. J. Neurol. Neurosurg. Psychiat. 32, 462-469 (1969)

15. Thomas, P.K.: Peripheral nerve function. Seminar on neurology of leprosy and peripheral neuropathy. U.S. Public Health Service Hospital. Carville, Louisiana. February 20-21, 1974

16. Eliasson, S.G.: Nerve conduction changes in experimental diabetes. J. clin. Invest. 43, 2353-2358 (1964)

17. Eliasson, S. G.: Properties of isolated nerve fibers from alloxanized rats. J. Neurol. Neurosurg. Psychiat. 32, $525-529(1969)$

18. Chopra, J.S., Hurwitz, L.J.: Sural nerve myelinated fiber density and size in diabetes. J. Neurol. Neurosurg. Psychiat. 32, 149-154 (1969)

19. Dolman, C.L.: The morbid anatomy of diabetic neuropathy. Neurology (Minneap.) 13, 135-142 (1963)

20. Greenbaum, D., Richardson, P.C., Salmon, M.V., Urich, H.: Pathological observations on six cases of diabetic neuropathy. Brain 87, 201-214 (1964)

21. Reske-Nielsen, E., Lundbaek, K. : Pathological changes in the central and peripheral nervous system of young long-term diabetics. Diabetologia 4, 34-43 (1968)

22. Chopra, J.S., Fannin, T.: Pathology of diabetic neuropathy. J. Path. Bact. 104, 175-184 (1971)

23. Budzilovich, G.N.: Diabetic neuropathy complex. Virchows Arch. path. Anat. 350, 105-122 (1970)

24. Appenzeller, O,, Richardson, E.P.: Sympathetic chain in patients with diabetic and alcoholic polyneuropathy. Neurology (Chic.) 16, 1203-1212 (1966)

25. Olsson, Y., Sourander, P.: Changes in the sympathetic nervous system in diabetes mellitus. J. Neuro-Visceral Relations 31, 86-95 (1968)

26. Woolf, A. L., Malins, J.: Changes in the neuromuscular nerve endings in diabetic neuropathy. A biopsy study. J. Path. Bact. 73, 316-317 (1957)

27. Cöers, E., Hildebrand, J.: Latent neuropathy in diabetes and alcoholism. Neurology (Chic.) 15, 19-38 (1965)

28. Locke, S., Lawrence, D.G., Legg, M.A.: Diabetic amyotrophy. Amer. J. Med. 34, 775-785 (1963)

29. Thomas, P.K., Lascelles, R.G.: The pathology of diabetic neuropathy. Quart. J. Med. 35, 489-509 (1966)

30. Chopra, J.S., Hurwitz, L.J., Montgomery, D.A.D.: The pathogenesis of sural nerve changes in diabetes mellitus. Brain 92, 391-418 (1969)

31. Cavanagh, J.B., Jacobs, J.M.: Some quantitative aspects of diphteritic neuropathy. Brit. J. exp. Path. $45,309-322$ (1964)

32. Jacobs, J.M., Cavanagh, J.B., Mellick, R.S.: Intraneural injection of diphtheria toxin. Brit. J. exp. Path. 47, 507-517 (1966)

33. Dyck, P.J., Johnson, W.J., Lambert, E.H., O'Brien, P.C.: Segmental demyelination secondary to axonal degeneration in uremic neuropathy. Proc. Mayo clin. 46, 400-423 (1971)

34. Thomas, P.K., Hollinrake, K., Lascelles, R.G., O'Sullivan, D.J., Daillod, R.A., Moorhead, J.F., MacKenzie, J.C.: The polyneuropathy of chronic renal failure. Brain 94, 761-780 (1971) 
35. Thomas, P.K.: The morphological basis for alterations in nerve conduction in peripheral neuropathy. Proc. roy. Soc. Med. 64, 295-298 (1971)

36. Ballin, R.H.M., Thomas, P.K. : Hypertrophic changes in diabetic neuropathy. Acta neuropath. (Berl.) 11, 93-102 (1968)

37. Vital, C., Vallat, J.M., LeBlanc, M., Martin, F., Coquet, M.: Etude ultrastructurale des neuropathies périphériques du diabète sucré. Nouv. Presse méd. 1, $2695-2698(1972)$
38. Asbury, A.K., Victor, M., Adams, R.D.: Uremic polyneuropathy. Arch. Neurol. (Chic.) 8, 413-428 (1963)

William W. Schlaepfer Department of Pathology

Washington Univ. Medical School

660 S. Euclid Ave.

St. Louis, Missouri, 63110 USA 